\title{
Structural Analysis of Micro-channels in Human Temporal Bone
}

\author{
Olivier Cros, Michael Gaihede, Mats Andersson and Hans Knutsson \\ Conference Publication
}

\section{Tweet}

N.B.: When citing this work, cite the original article.

Original Publication:

Olivier Cros, Michael Gaihede, Mats Andersson and Hans Knutsson, Structual Analysis of Micro-channels in Human Temporal Bone, IEEE 12th International Symposium on Biomedical Imaging (ISBI), 2015 IEEE 12th International Symposium on, 2015. (), pp.9-12.

http://dx.doi.org/10.1109/ISBI.2015.7163804

Copyright:www.ieee.org

Postprint available at: Linköping University Electronic Press

http://urn.kb.se/resolve?urn=urn:nbn:se:liu:diva-122177

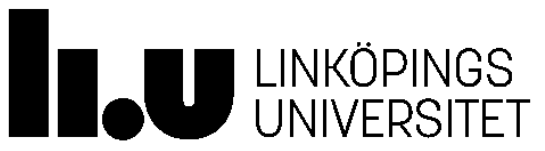




\title{
STRUCTURAL ANALYSIS OF MICRO-CHANNELS IN HUMAN TEMPORAL BONE.
}

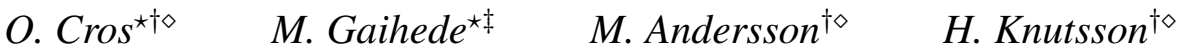 \\ $\dagger$ Department of Biomedical Engineering, Linköping University, Sweden \\ $\diamond$ Center for Medical Image Science and Visualization (CMIV), Linköping University, Sweden \\ * Department of Otolaryngology, Head \& Neck Surgery, Aalborg University Hospital, Denmark \\ $\ddagger$ Department of Clinical Medicine, Aalborg University, Denmark
}

\begin{abstract}
Recently, numerous micro-channels have been discovered in the human temporal bone by micro-CT-scanning. Preliminary structure of these channels has suggested they contain a new separate blood supply for the mucosa of the mastoid air cells, which may have important functional implications. This paper proposes a structural analysis of the microchannels to corroborate this role. A local structure tensor is first estimated. The eigenvalues obtained from the estimated local structure tensor were then used to build probability maps representing planar, tubular, and isotropic tensor types. Each tensor type was assigned a respective RGB color and the full structure tensor was rendered along with the original data. Such structural analysis provides new and relevant information about the micro-channels but also their connections to mastoid air cells. Before carrying a future statistical analysis, a more accurate representation of the micro-channels in terms of local structure tensor analysis using adaptive filtering is needed.
\end{abstract}

Index Terms - human temporal bone, mastoid, microchannels, quadrature filters, structure tensor, visualization

\section{INTRODUCTION}

A recent study using micro-CT scans of human temporal bone specimens has revealed the existence of multiple microchannels carved in the bone surrounding the mastoid air cell system [1]. The structure and size of these micro-channels resemble a vascular network which points to a new and separate blood supply for the mucosa of the mastoid air cell system [1]. This observation corroborates with current ideas on an active function of the mucosa where the physiological regulation of the middle ear pressure is determined by the vascular congestion of the mucosa [2]. In clinical otology the overall pressure regulation is of immense importance, and hitherto, the mastoid air cell system has only been attributed a passive role [3].

However, the effects of changes in vascular congestion depend on a rich blood supply which may be provided by

This research has been supported by The Obel Family Foundation. a vascular network in these micro-channels. This new potential role may be strengthened further by a detailed structural analysis of the micro-channels, where their similarities with a vascular network are qualitatively investigated together with their communication with the mucosa at the surface of the mastoid air cells. The micro-channels resemble tubular-like

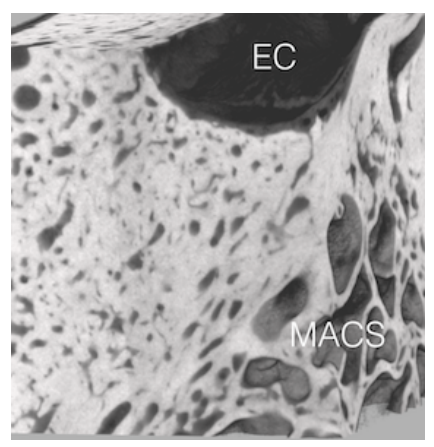

(a)

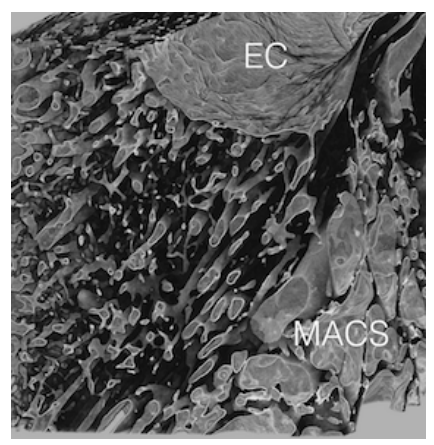

(b)
Fig. 1. Volume rendering of a cropped micro-CT scanning of a human temporal bone using a (a): ramp transfer function, and using (b) trapezoidal transfer function. EC: ear canal, MACS: mastoid air cell system.

structures and appear to have a wide range of shapes and diameters [1]. This resembles to a vascular network and could lead to the use of existing vessel segmentation methods [4]. However, observations based on histological sections have revealed the presence of both arterioles and venules inside. Therefore, their structural content also needs to be taken into consideration.

Local structure tensor analysis based on a second order tensor with six degrees of freedom in 3D may be a more robust representation of the channels [5]. Many versions of the local structure tensor analysis have been used for specific needs, especially for image enhancement via adaptive filtering, [6]. Thus, this paper presents a structural pre-analysis of the micro-channels from the human temporal bone by using local structure tensor analysis based on micro-CT scanning. 


\section{MATERIAL}

One human temporal bone specimen was used in this study. The bone specimen was scanned at the Department of Physics and Astronomy, Ghent, Belgium, using the same procedure as in [1,7]. The resolution of the scans is $32 \mu \mathrm{m}$ isotropic. The micro-CT scan was cropped from an original size of $1820 \times 1820 \times 1211$ voxels down to a limited size of $690 \times 490 \times 480$ voxels. This test volume was chosen to represent micro-channels at the level of the ear canal (EC) and in the proximity of mastoid air cell system (MACS) with a large variety of sizes and shapes. The data is partially represented on Figure 1a using volume rendering with a default ramp transfer function (TF) [8]. A better representation of the micro-channels is possible using a trapezoidal transfer function by only selecting the voxels representing the bone surface [8]. Figure $1 b$ is used as the reference image.

\section{METHODS}

Finding a good representation of the micro-channels is of primary importance. Such structures can be represented using a structure tensor by its corresponding eigenvalues and eigenvectors.

$$
\mathbf{T}=\sum_{k=1}^{3} \lambda_{k} \hat{\mathbf{e}}_{k} \hat{\mathbf{e}}_{k}^{T} \quad \text { with } \quad \lambda_{k}>\lambda_{k+1} \geq 0
$$

Three particular cases of the structure tensor where $\lambda_{1} \geq$ $\lambda_{2} \geq \lambda_{3} \geq 0$ are the eigenvalues in decreasing order, $\hat{e}_{i}$ the eigenvector corresponding to $\lambda_{i}$, and where $\mathbf{I}$ is the identity matrix $\mathbf{I}=\hat{\mathbf{e}}_{1} \hat{\mathbf{e}}_{1}^{T}+\hat{\mathbf{e}}_{2} \hat{\mathbf{e}}_{2}^{T}+\hat{\mathbf{e}}_{3} \hat{\mathbf{e}}_{3}^{T}$, can be extracted as:

- $\lambda_{1}>0, \quad \lambda_{2}=\lambda_{3}=0, \quad \mathbf{T}_{1}=\lambda_{1} \hat{\mathbf{e}}_{1} \hat{\mathbf{e}}_{1}^{T}$. This case corresponds to a neighborhood that is perfectly planar, i.e. is constant on planes in a given orientation. The orientation of the normal vectors to the planes is given by $\hat{e}_{1}$. Edges from large structures are represented through this rank 1 tensor.

- $\lambda_{1}=\lambda_{2}>0, \quad \lambda_{3}=0, \quad \mathbf{T}_{2}=\lambda_{1}\left(\mathbf{I}-\hat{\mathbf{e}}_{3} \hat{\mathbf{e}}_{3}^{T}\right)$. This case corresponds to a neighborhood that is constant on lines and/or on tubular structures. The orientation of the lines is given by the eigenvector corresponding to the least eigenvalue, $\hat{e}_{3}$. The micro-channels are represented through this rank 2 tensor.

- $\lambda_{1}=\lambda_{2}=\lambda_{3}>0, \quad \mathbf{T}_{3}=\lambda_{1} \mathbf{I}$. This case corresponds to an isotropic neighborhood, meaning that there exists energy without any specific orientation in the neighborhood, e.g. in the case of noise. This case is a rank 3 tensor.

The eigenvalues from the structure tensor can further be used to estimate the probability of each visited neighborhood belonging to either a rank 1 , rank 2 , or a rank 3 tensor, defined by $p_{1}, p_{2}$, and $p_{3}$, where $\sum_{k=1}^{3} p_{k}=1$ which can be seen as probabilities as [5].

$$
p_{1}=\frac{\lambda_{1}-\lambda_{2}}{\lambda_{1}}, \quad p_{2}=\frac{\lambda_{2}-\lambda_{3}}{\lambda_{1}}, \quad p_{3}=\frac{\lambda_{3}}{\lambda_{1}}
$$

Natural structures like the micro-channels are however composed of a mixture of these three cases and a more general ideal structure tensor should instead be represented as

$$
\mathbf{T}=p_{1} \mathbf{T}_{1}+p_{2} \mathbf{T}_{2}+p_{3} \mathbf{T}_{3}
$$

To estimate a structure tensor based on the tensor equation given in Eq.3, a set of quadrature filter responses were computed over the whole data volume. These complex-valued quadrature filters are used to pick up local energy in several directions in the Fourier space. The advantage of using quadrature approach is its phase-independence property where the response of the quadrature filter will be equally strong for odd and even structures, which will either represent a microchannel as a line or locally as an edge depending on the size of the micro-channel being processed and the scale being used. A typical frequency function used as quadrature filter is given below

$$
F_{k}(\hat{\mathbf{u}})=R(\rho) D_{k}(\hat{\mathbf{u}})
$$

To pick up energy in all possible orientations, the quadrature filter is built as spherically separable in the Fourier domain with an arbitrary but positive radial bandwidth function $R(\rho)$, with $\rho=\|\hat{\mathbf{u}}\|$, and a direction function $D_{k}(\hat{\mathbf{u}}) . R(\rho)$ is typically designed as a bandpass function based on a center frequency and a bandwidth. It determines in what scale the filter has its sensitivity. The direction function varies as $\cos ^{2}(\phi)$ where $\phi$ is the difference between $\mathbf{u}$ and the filter direction $\mathbf{n}_{k}$ and given by

$$
D_{k}(\mathbf{u})= \begin{cases}\left(\hat{\mathbf{u}} \cdot \hat{\mathbf{n}}_{k}\right)^{2} & \text { if }\left(\hat{\mathbf{u}} \cdot \hat{\mathbf{n}}_{k}\right) \geq 0 \\ 0 & \text { otherwise }\end{cases}
$$

where $\hat{\mathbf{n}}_{k}$ are the directions in the Fourier space where each quadrature filter picks up an energy from the local neighborhood. The magnitude of the quadrature filter output is then computed

$$
q_{k}=\left\|\frac{1}{2 \pi} \int S(\mathbf{u}) F_{k}(\mathbf{u}) d \mathbf{u}\right\|
$$

and the estimate of the structure tensor can now be constructed as

$$
\mathbf{T}_{e s t}=\sum_{k=1}^{6} q_{k}\left(\alpha \hat{\mathbf{n}}_{k} \hat{\mathbf{n}}_{k}^{T}-\beta \mathbf{I}\right)
$$

where $q_{k}$ represents the magnitude of the filter output, $\hat{\mathbf{n}}_{k}$ the orientation of the quadrature filter with direction $k$, which is 6 in 3D. For 3D, $\alpha=\frac{5}{4}$ and $\beta=\frac{1}{4}$. To assess the resulting $p_{1}, p_{2}$, and $p_{3}$ from the estimated structure tensor, we used volume rendering through MeVisLab, a free medical image processing and visualization software. Because, $p_{1}, p_{2}$, and 
$p_{3}$ are probability measures of each special case, we wanted to have a more natural span going from rank 1 tensor to rank 2 tensor with the rank 3 tensor in between so as to move from a tubular structure towards a planar structure via an isotropic structure. To visualize this natural transition between the three cases, a lookup table (LUT) was created where each RGBA channel corresponds a specific case, namely the rank 2 tensor was assigned the red color, the rank 3 tensor was assigned the green color, and the rank 1 tensor was assigned the blue color. To use the LUT function in MeVisLab, each RGB channel was controlled by a respective non-linear function based on sigmoid functions. The 3D volume representing all the special cases was created as

$$
p_{m}=p_{1}+\gamma p_{3}
$$

with $\gamma=0.4$, leading to $p_{m}=1$ for rank 1 tensor, $p_{m}=0$ for rank 2 tensor, and $p_{m}=0.4$ for rank 3 tensor. This mapping was built to fit the RGB channels. Because micro-channels present variations in terms of rank 1 and rank 2 tensor types, the $\alpha$-channel was so that rank 1 and rank 2 tensor types were fully visible while the rank 3 tensor type was partly set transparent so as to allow a certain degree of mixtures between the three special cases. The LUT is illustrated in Figure 2. To

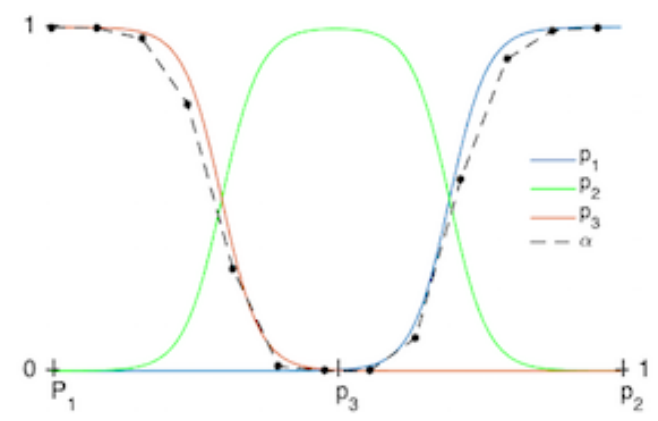

Fig. 2. RGB LUT representing the probability function $p_{m}$.

interactively adjust the settings while visualizing the results, a MeVisLab node called LutEditor was used to move some control points to decide the structures to look at and which structure should be visible or made transparent.

\section{RESULTS AND DISCUSSION}

Before presenting and discussing the results, it should be noted that the local structure tensor analysis does not inform about the specific tissue type, rather a description of how planar, tubular, or isotropic a local structure is. Figure 3a illustrates all structures at once from the volume $p_{m}$. Figure $3 \mathrm{~b}-\mathrm{c}$ respectively represents $p_{1}, p_{2}$, and $p_{3}$ with their corresponding RGB color channel and helps to better understand their complimentary contribution. Overlaying $p_{1}, p_{2}$, and $p_{3}$ on the original data further informs about their locations with respect to the bone structures, i.e. within the micro-channels, the ear

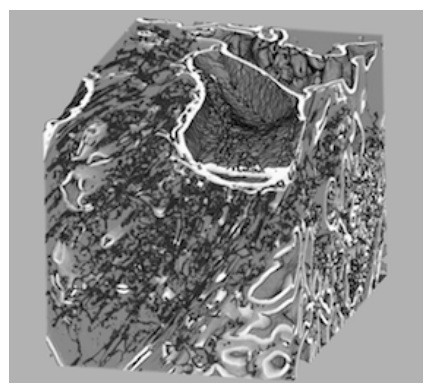

(a) $p_{m}$

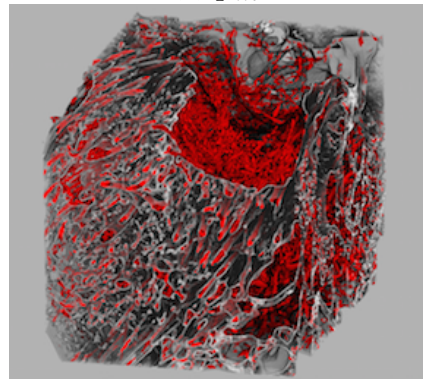

(c) Rank 2 tensor

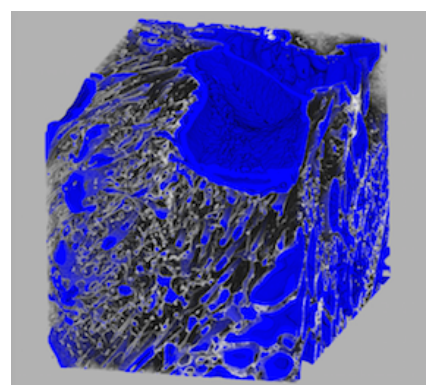

(b) Rank 1 tensor

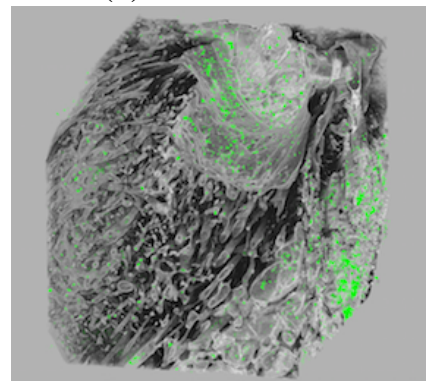

(d) Rank 3 tensor
Fig. 3. (a): Mixture of all three cases into the single volume $p_{m}$. (b): $p_{1}$ representing planar structures as blue. (c): $p_{2}$ representing line and tubular structures as red. (d): $p_{3}$ representing isotropic structures as green.

canal (EC), or within the mastoid air cells. Where Figure 3b-d represents each case separately, Figure 4 is based on the proposed LUT giving a more natural transition between the different structures. Figure 4 gives a non-exhaustive representation of micro-channels with various different shapes encountered during the analysis. Figure 4A illustrates the perfect rank 2 tensor type structures within the micro-channels. Figure 4B emphasizes the transition from a rank 1 tensor type structure to a rank 2 tensor type structure. Figure $4 \mathrm{C}$ illustrates the in-

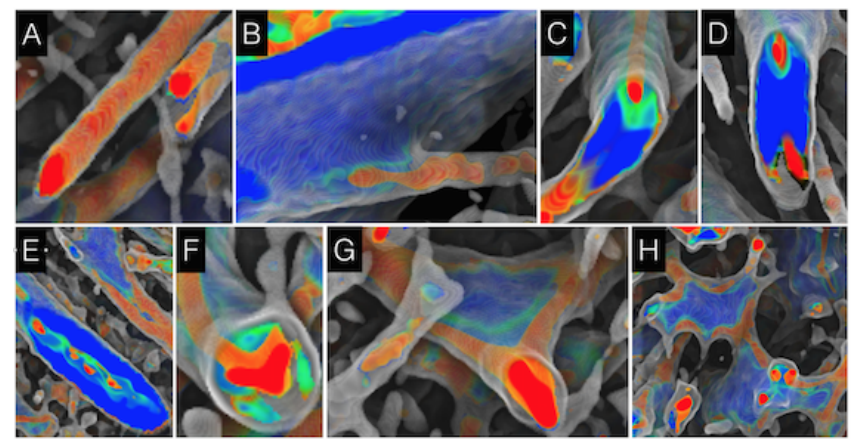

Fig. 4. Micro-channels with different shapes.

termix within a micro-channel of $p_{1}, p_{2}$ tensor type structures with occasionally a slight amount of $p_{3}$ tensor type structure. Figure 4D depicts a broader micro-channel with two rank 2 tensor type structures placed on either side of a micro-channel 
with a well-defined rank 1 tensor type structure filling the gap in between. Figure 4E reveals a flatten micro-channel with seemingly several rank 2 tensor type structures in the central part, and surrounded by a rank 1 tensor type structure. Figure $4 \mathrm{~F}$ pictures the merging of two to three rank 2 tensor type structures into a larger rank 2 tensor type structure at the center of a larger micro-channel.

Figure $4 \mathrm{G}$ presents a structure resembling a hub, never described before, receiving three micro-channels at its extremities with a rank 2 tensor type structure running along the edges and with a rank 1 tensor type structure at its centre. Figure $4 \mathrm{H}$ describes a similar hub structure having more connections. As for the hub, presence of more than one rank 2 tensor type structures in a single micro-channel along with bifurcation or merging of rank 2 tensor type structures have never been reported in the literature before. Figure 5 depicts

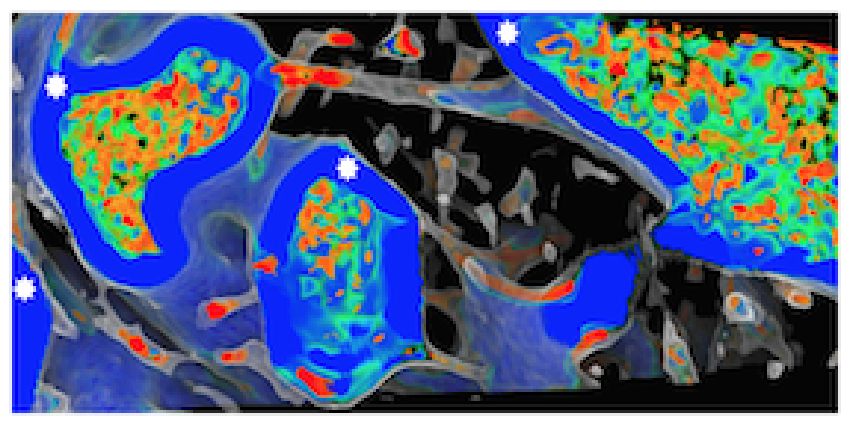

Fig. 5. Micro-channels connecting to mastoid air cells (white stars).

several mastoid air cells partially cut (white stars). The second mastoid air cell, to the left, connects on its right side to a micro-channel with a net transition from rank 1 tensor type to a rank 2 tensor type. On the opposite side of this microchannel, the rank 1 tensor type structure lining the wall of the mastoid air cell (star to the right) penetrates the channel. The end-tip of the rank 1 tensor structure type indentation displays a transition toward a rank 2 tensor type structure, strongly indicating a red rank 2 tensor type structure in the missing part of the channel. The same applies to the lower right part of the middle mastoid air cell where two micro-channels clearly connect to a hub structure.

Where the weak structures present in $p_{1}, p_{2}$, and $p_{3}$ could be reduced, the use of the proposed LUT did not allow this filtering, and therefore the presence of mixture of $p_{1}, p_{2}, p_{3}$ tensor types structures is visible in the mastoid air cells. Detection of weak structures illustrates the fact that structures inside the micro-channels can be smaller than the noise visible in the mastoid air cells. A tradeoff between the full detection of fine structures within the small micro-channels and the amount of noise to filter out is therefore necessary.

\section{CONCLUSION}

This study has demonstrated the structural variation of contents inside the micro-channels by a local structure tensor analysis. From this analysis, discovery of unreported hub structures may help understand the origin and possible multi-role of this complex network formed by these micro-channels. Presence of noise within the air cells along with the missing information in some micro-channels suggest the future need of image enhancement using an adaptive filtering technique based on the local structure tensor analysis used in this study. A larger scale study is also considered in the future in order to validate the method proposed in this pre-analysis.

\section{REFERENCES}

[1] O. Cros, M. Borga, E. Pauwels, J.J.J. Dirckx, and M. Gaihede, "Micro-channels in the mastoid anatomy. indications of a separate blood supply of the air cell system mucosa by micro-ct scanning.," Hearing Research, vol. 301, pp. 60-65, 2013.

[2] M. Gaihede, O. Cros, and S. Padurariu, "The role of the mastoid in middle ear pressure regulation," In Takahashi $H($ Ed.) "Cholesteatoma and Ear Surgery - An Update". Proceedings of the 9th International Conference on Cholesteatoma and Ear Surgery, pp. 17-20, 2012.

[3] J.J.J. Dirckx, Y. Marcusohn, M. Gaihede, S. Puria, R.R. Fay, and A.N. Popper, Quasi-static Pressures in the Middle Ear Cleft, pp. 93-133, Springer Handbook of Auditory Research. Springer New York, 2013.

[4] D. Lesage, E. Angelini, I. Bloch, and G. Funka-Lea., "A review of $3 \mathrm{~d}$ vessel lumen segmentation techniques: models, features and extraction schemes.," Medical Image Analysis, vol. 13(6), pp. 819-845, 2009.

[5] G. Granlund and H. Knutsson, Signal Processing for Computer Vision., Kluwer Academic Publishers, Dordrecht, 1995.

[6] A. Eklund, M. Andersson, and H. Knutsson, "True 4D image denoising on the GPU.," International Journal of Biomedical Imaging, vol. 2011, pp. 1-16, 2011.

[7] B.C. Masschaele, V. Cnudde, M. Dierick, P. Jacobs, L. Van Hoorebeke, and J. Vlassenbroeck, "UGCT: new X-ray radiography and tomography facility.," Nucl. Instrum. Methods Phys. Res., vol. A 580, pp. 266-269, 2007.

[8] D. R. Ney, E. K. Fishman, D. Magid, and R. A. Drebin, "Volumetric rendering of computed tomography data: principles and techniques," Computer Graphics and Applications, IEEE, vol. 10, no. 2, pp. 24-32, 1990. 